\title{
New closure method for a simultaneous diazo coupling between 1- and 2-Naphthols and diazotized sulphanilic acid
}

\author{
Jerzy Bałdyga, Magdalena Jasińska \\ Warsaw University of Technology, Faculty of Chemical and Process Engineering, ul. Waryńskiego 1, 00-645 Warszawa, \\ Poland, e-mail: baldyga@ichip.pw.edu.pl
}

\begin{abstract}
This paper presents the effects of mixing on the course of complex chemical reactions in relation to the manufacturing of pure products at high reaction selectivity, and designing mixing strategies using complex, homogeneous test reaction systems. As an example, the competitive-consecutive and parallel reaction test system including a simultaneous diazo-coupling between 1- and 2-naphtols and diazotized sulphanilic acid is considered. The effect of mixing on the reaction selectivity is interpreted using mechanistic modeling and a new closure scheme. The new closure represents an extension of the conditional PDF closure applied before to simple reaction schemes.
\end{abstract}

Keywords: closure method, diazo-coupling, micromixing, turbulence.

\section{INTRODUCTION}

In industry, many homogeneous chemical reactions leading to desirable intermediate and end-products are accompanied by side reactions producing undesired by-products. By-products decrease the reaction yield and complicate the product separation. To improve the selectivity, the competition between reactions can be enhanced; one can either add a homogeneous catalyst that increases the rate of a desired reaction or an inhibitor that decreases the rate of side reactions. This creates again separation problems; moreover, when the rate constant of the desired reaction is increased, its rate becomes controlled by mixing, rather than by the reaction kinetics. Competition is then between the mixing-controlled desired reaction and the slower side-reactions ${ }^{1}$. In any case the problem of „reactive mixing” arises. Understanding of this problem and development of micromixing models that enable to predict the effects of mixing on selectivity helps to reduce the amount of undesired by-products and avoid separation. The modelling problem will be solved in this work with the use of two methods: the first one is based on the already published mechanistic modelling method ${ }^{\mathbf{1}}$ and the other one is based on a closure scheme. The closure scheme will be based on the conditional PDF closure. A similar closure method was applied previously to simple reactions $\mathbf{s}^{\mathbf{1}, 2}$.

Multiple chemical reactions also offer the best way to test the mixing models and characterize experimentally the mixing performance in the equipment of industrial importance ${ }^{1}$. In the present case a simultaneous diazocoupling between 1- and 2-naphtols and diazotized sulphanilic acid is considered. This reaction scheme was applied previously to characterize the experimental mixing in the stirred-tank reactor ${ }^{1}$, the static mixer ${ }^{1}$, the rotorstator mixer ${ }^{3}$, and the microreactor. The reaction scheme contains an irreversible parallel and consecutive second order reactions:

$$
\begin{array}{ll}
A_{1}+B \stackrel{k_{1 p}}{\longrightarrow} p-R & A_{1}+B \stackrel{k_{1 o}}{\longrightarrow} o-R \\
p-R+B \stackrel{k_{2 o}}{\longrightarrow} S & o-R+B \stackrel{k_{2 p}}{\longrightarrow} S \\
A_{2}+B \stackrel{k_{3}}{\longrightarrow} Q &
\end{array}
$$

where $A_{1}$ denotes 1 -naphtol, $A_{2}$ is 2-naphtol, $B$ represents diazotized sulphanilic acid, $o-R$ and $p-R$ are two monosubstituted dyes (ortho and para), $S$ represents a bisazo dye and $Q$ is a single monoazo dye. At the temperature equal to $298 \mathrm{~K}, \mathrm{pH}=9.9$ (with sodium carbonate and bicarbonate employed as buffers), for the ionic strength $\mathrm{I}=444.4 \mathrm{~mol} / \mathrm{m}^{3}$ the rate constants take the following values as given by (Bourne et al. $)^{4}: k_{l p}=12238 \pm 446$ $\mathrm{m}^{3} /(\mathrm{mol} \mathrm{s}), k_{1 o}=921 \pm 31 \mathrm{~m}^{3} /(\mathrm{mol} \mathrm{s}), k_{2 p}=22.25 \pm$ $0.25 \mathrm{~m}^{3} /(\mathrm{mol} \mathrm{s}), k_{2 o}=1.835 \pm 0.018 \mathrm{~m}^{3} /(\mathrm{mol} \mathrm{s}), k_{3}=$ $124.5 \pm 1.0 \mathrm{~m}^{3} /(\mathrm{mol} \mathrm{s})$.

The extent of mixing is characterized by the product distribution. Two measures of product distribution can be applied, the first one representing the yield of the secondary product $\mathrm{S}$ and the second one the yield of the competitive product $Q^{1}$.

$$
\begin{aligned}
& X_{S}=2 c_{S}\left(c_{o R}+c_{p R}+c_{Q}+2 c_{S}\right) \\
& X_{Q}=c_{Q}\left(c_{o R}+c_{p R}+c_{Q}+2 c_{S}\right)
\end{aligned}
$$

Both definitions express the yields relative to the limiting reactant $B$. They represent the product distribution, provided that the limiting reactant (diazotized sulphanilic acid) had been completely consumed. Hence, they represent the fraction of $B$ transformed either to bisazo dye $\mathrm{S}$ or to monoazo dye $\mathrm{Q}$, respectively. Under most operating conditions $X_{S}$ and $X_{O}$, decrease as the mixing rate increases. Considering the relations of the product distribution to the reaction rate constants, $X_{S}$ is usally applied as a measure of less intensive mixing, whereas $X_{Q}$ represents at the same time a good measure of intensive mixing. To show the limiting values of $X_{S}$ and $X_{Q}$, and observe in this way a range of product distribution variation with mixing, one should consider two distinct regimes of mixing:

a) A slow reaction regime. Here we consider the product distribution in the chemical regime (or instantaneous mixing regime relative to reaction rate). In this regime the stoichiometric ratios and the chemical kinetics determine the product distribution. In practise, with increasing the rate of kinetic energy dissipation, $\varepsilon$, and increasing in this way the mixing intensity, $X_{S}$ and $X_{Q}$ show the tendency towards asymptotic values, which no longer depend on the rate of energy dissipation, $\varepsilon$ but which can be calculated 
directly from the reaction kinetics and the stoichiometric ratio values. Very little of bisazo dye is formed, especially $\mathrm{S}$. In the chemical regime the concentration of naphtols and the resulting selectivity can be calculated from equations (8), (9) and $(10)^{\mathbf{1}, 4}$.

$x^{\mathrm{\kappa}}+\xi x=1+\xi-1 / \gamma \quad$ and $\quad \xi z=x^{\mathrm{\kappa}}$

$X_{Q}=(1-x) /(1 / \xi+1-x-z)$ and $X_{S}=0$

where $x=c_{A 2} / c_{A 2,0}, \quad z=c_{A 1} / c_{A 2,0}, \kappa=\left(k_{1 p}+k_{10}\right) / k_{3}$; $\gamma=N_{A 1,0} / N_{B, 0}$ represents the stoichiometric ratio of 1-naphthol $\left(\mathrm{A}_{1}\right)$ to diazonium ion (B) and $\xi=N_{A 2,0} / N_{A 1,0}$ is the stoichiometric ratio of 2-naphthol to 1-naphthol.

b) An instantaneous reaction regime. Here we consider the product distribution in the fully mixing controlled regime (or very slow mixing regime). In this case the mixing rate is very low relative to the possible reaction rate so complete segregation of reactants occurs, and the product distribution attains another asymptotic value, where $X_{S}$ and $X_{Q}$ become again independent of both mixing and the related rate of energy dissipation, $\varepsilon$. When all the reactions are instantaneous relative to mixing then the intermediates o-R and $\mathrm{p}-\mathrm{R}$ are fully converted to $\mathrm{S}$, whilst $\mathrm{A}_{2}$ is partially converted to $\mathrm{Q}$.

$X_{Q}=\xi /(\xi+2), X_{S}=1-X_{Q}$

Figure 1 shows an effect of stoichiometric ratio, $\xi=N_{A 2,0} / N_{A 1,0}$, on the limiting values of $X_{S}$ and $X_{Q}$; one can see that at slow mixing $X_{S}$ characterizes its intensity, and then small values of $\xi$ should be used. When mixing is fast and thus $X_{Q}$ is applicable, then larger values of $\xi$ should be used.

To model effects of mixing on various scales on the course of chemical reactions, a non-equilibrium multipletime-scale mixing model and a beta distribution of the mixture fraction will be applied in combination with a conditional moment closure based on the linear interpolation of local instantaneous reactant concentration values. As mentioned above this closure method was applied previously to simple reaction schemes ${ }^{\mathbf{1 , 2}}$.

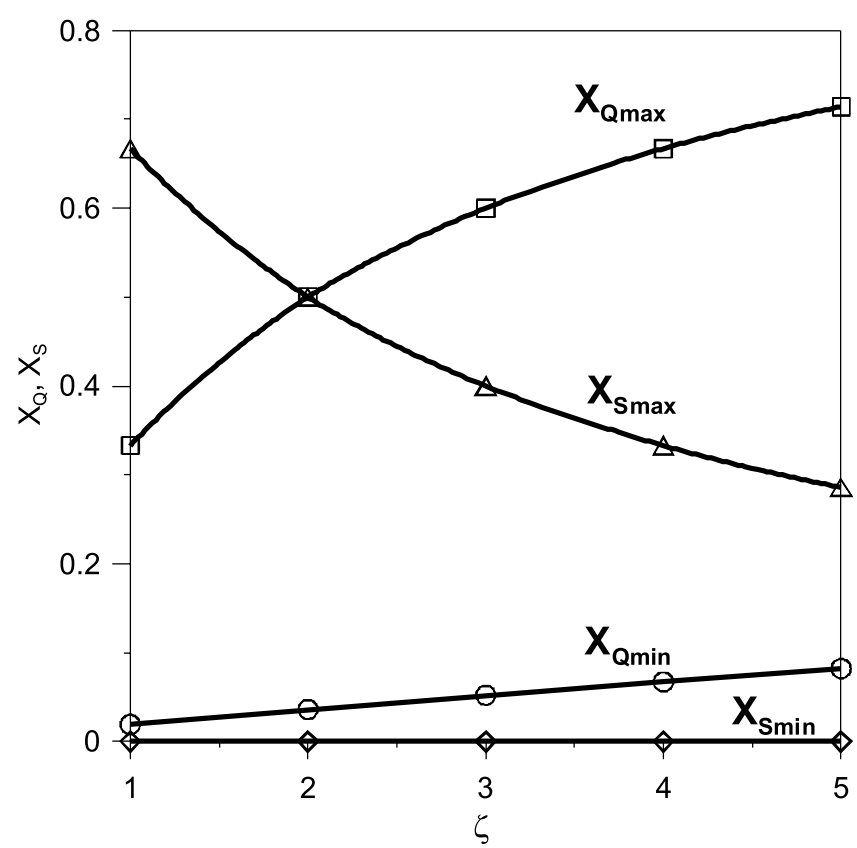

Figure 1. The effect of stoichiometric ratio, $\xi$, on limiting values of $X_{Q}$ and $X_{S}$ for $\gamma=N_{A 1} / N_{B}=1.2$ and $\kappa=106$

\section{MODELLING OF MIXING EFFECTS ON THE COURSE OF DIAZO-COUPLING REACTIONS}

The conclusions drawn out from the results depicted in Fig. 1 can be confirmed by applying a relatively simple engulfment model of micromixing ${ }^{1}$. When micromixing is controlled by viscous-convective engulfment effects, then the concentration history can be calculated from the engulfment equation:

$d c_{i} / d t=E\left(\left\langle c_{i}\right\rangle-c_{i}\right)+r_{i}$

where $E=0.058(\varepsilon / v)^{0.5}$ represents the engulfment parameter, determining the rate of mixing on the molecular scale, and $r_{i}$ is the rate of reaction. Typical model predictions are shown in Figure 2 ab.

a)

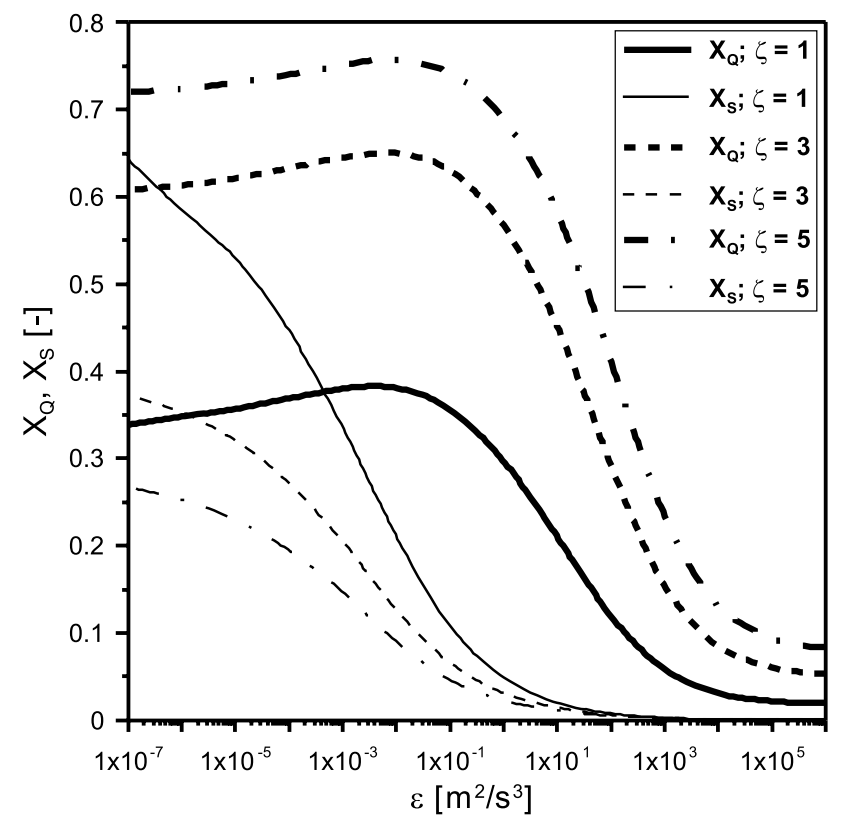

b)

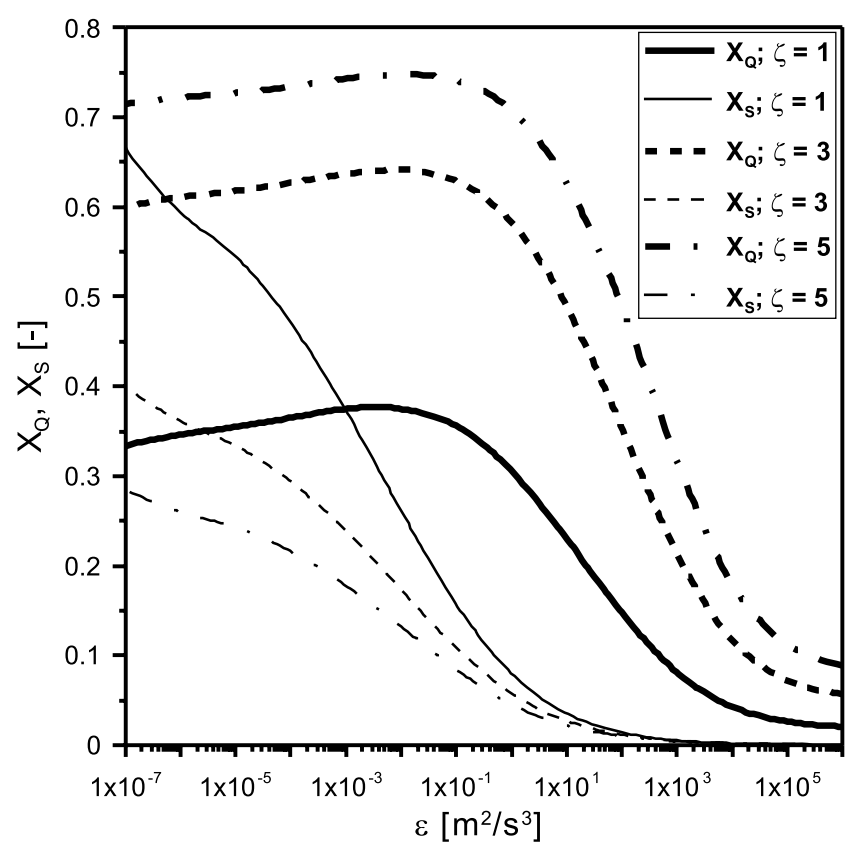

Figure 2. The effect of the rate of energy dissipation and stoichimetric ratio, $\xi$, on the product distribution for $\mathrm{c}_{\mathrm{B} 0, \mathrm{sr}}=0.5 \mathrm{~mol} / \mathrm{m}^{3}, \mathrm{c}_{\mathrm{A} 10, \mathrm{sr}}=0.6 \mathrm{~mol} / \mathrm{m}^{3}$ (a) $\alpha=V_{A} / V_{B}=20$, (b) $\alpha=V_{A} / V_{B}=100$ 
Clearly $X_{S}$ is sensitive to mixing at small energy input and $X_{Q}$ becomes mixing sensitive at higher energy input values.

Let us consider now the method based on the probability distribution function (PDF). The PDF method applied in what follows is based on the probability density function of the passive scalar concentration distribution; the passive scalar concentration is normalized and expressed by the mixture fraction, $f$, (Bałdyga, Bourne) ${ }^{\mathbf{1}}$ :

$f=c_{A}^{0} / c_{A 0}=c_{A 1}^{0} / c_{A 10}=c_{A 2}^{0} / c_{A 20}=1-c_{B}^{0} / c_{B 0}$

In what follows the beta probability density function is applied. The beta distribution has been recommended by many authors ${ }^{1}$; it is U-shaped for early stages of mixing and thus at high values of the intensity of segregation $I_{S}$, and is bell-shaped for the final stages of mixing and thus at small values of $I_{S}, I_{S}=\operatorname{var} f /[\operatorname{var} f]_{0}$.

$\Phi(f)=f^{v-1}(1-f)^{w-1} / B(v, w) ; \quad B(v, w)=\int_{0}^{1} x^{v-1}(1-x)^{w-1} d x$

where $v=\bar{f}\left(1-I_{S}\right) / I_{S}, w=(1-\bar{f})\left(1-I_{S}\right) / I_{S}$. For mixing simulated using the engulfment model and constant value of the rate of energy dissipation the intensity of segregation decreases exponentially $I_{s}=\exp (-E t)$.

The closure is based on the linear interpolation of concentrations between the limits for very slow and instantaneous reactions:

$c_{i}(f)=c_{i}^{\infty}(f)+\left[c_{i}^{0}(f)-c_{i}^{\infty}(f)\right] \overline{\overline{c_{i}}}-\overline{c_{i}^{\infty}} \overline{\overline{c_{i}^{0}}-\overline{c_{i}^{\infty}}}$

The limiting values for very slow reactions are given by eq.(14), and for instantaneous reactions by eqs (17), (18) and (19).

$C_{A 1}^{\infty}(f)=C_{A 2}^{\infty}(f)=1-f\left[c_{B 0}+(1+\beta) c_{A 10}+c_{A 20}\right] /$

$/\left[(1+\beta) c_{A 10}+c_{A 20}\right]$ for $f<f_{s}$

$C_{B}^{\infty}(f)=f\left[c_{B 0}+(1+\beta) c_{A 10}+c_{A 20}\right] / c_{B 0}-$

$-\left[(1+\beta) c_{A 10}+c_{A 20}\right] / c_{B 0}$ for $f \geq f_{s}$

and $C_{A 1}^{\infty}(f)=C_{A 2}^{\infty}(f)=0, C_{B}^{\infty}(f)=0$ otherwise,

$C_{R}^{\infty}(f)=\frac{(1-f)-C_{A l}^{\infty}(f)}{\left(\frac{\beta}{1-\beta}+1\right)}=\frac{(1-f)-C_{A 2}^{\infty}(f)}{\left(\frac{\beta}{1-\beta}+1\right)}$

where $\beta=\frac{\overline{c_{S}}}{\overline{c_{S}}+\overline{c_{R}}}$ and $f_{s}=\frac{(1+\beta) c_{A 10}+c_{A 20}}{c_{B 0}+(1+\beta) c_{A 10}+c_{A 20}}$.

Eqs (17) and (18) are based on an observation that the probability distribution of an inert-type composition variable formed with a difference of reactant concentrations for instantaneous reaction behaves like the mixture fraction $f$ given by eq. (14) $)^{1}$.

In the regime of instantaneous reaction the set of eqs $(1) \div(5)$ can be replaced by

$A_{1}+2 B \stackrel{r_{i \infty}}{\longrightarrow} S$

$A_{2}+B \stackrel{r_{2 \infty}}{\longrightarrow} Q$

and the mixture fraction, $f$, can be expressed by:

$f=\frac{2 c_{A 1}^{\infty}+c_{A 2}^{\infty}-c_{B}^{\infty}+c_{B 0}}{2 c_{A 10}+c_{A 20}+c_{B 0}}$
However, during the real process one has also some $\mathrm{o}-\mathrm{R}$ and $\mathrm{p}-\mathrm{R}$ accumulated. For any moment one has $\overline{c_{R}}=\overline{c_{o R}}+\overline{c_{p R}}>0$. Then instead of eqs (20), (21) one can write:

$A_{1}+(1+\beta) B \stackrel{r_{1 \infty}}{\longrightarrow}(1-\beta) R+\beta S$

$A_{2}+B \stackrel{r_{2 \infty}}{\longrightarrow} Q$

where $(1-\beta)$ represents the amount of B already converted to $\mathrm{R}$.

Application of eqs (23), (24) leads to a new definition of the mixture fraction variable:

$f=\frac{(1+\beta) c_{A 1}^{\infty}+c_{A 2}^{\infty}-c_{B}^{\infty}+c_{B 0}}{(1+\beta) c_{A 10}+c_{A 20}+c_{B 0}}$

In the regime of instantaneous reactions reactants $\mathrm{A}_{1}$, $\mathrm{A}_{2}$ and $\mathrm{B}$ cannot coexist, which leads to eqs (17) and (18); notice that in this regime one has $c_{A 1}^{\infty} / c_{A 10}=c_{A 2}^{\infty} / c_{A 20}=C_{A 1}^{\infty}=C_{A 2}^{\infty}$. A similar approach leads to eq. (19).

Average concentrations are calculated at any point in the system by integrating over all fluid elements marked by $f$ values.

$\overline{c_{i}}=\int_{0}^{1} c_{i}(f) \Phi(f) d f$

This leads to the following set of equations describing Reynolds averaged reaction kinetics in a turbulent flow in the batch or the plug flow system:

$\frac{d \overline{C_{A 1}}}{d t}=-c_{B 0}\left(k_{1 p}+k_{1 o}\right) \int_{0}^{1} C_{A 1}(f) C_{B}(f) \Phi(f) d f$

$\frac{d \overline{C_{A 2}}}{d t}=-k_{3} c_{B 0} \int_{0}^{1} C_{A 2}(f) C_{B}(f) \Phi(f) d f$

$\frac{d \overline{C_{p R}}}{d t}=k_{1 p} c_{B 0} \int_{0}^{1} C_{A 1}(f) C_{B}(f) \Phi(f) d f-$

$-k_{2 o} c_{B 0} \int_{0}^{1} C_{p R}(f) C_{B}(f) \Phi(f) d f$

$\frac{d \overline{C_{o R}}}{d t}=k_{1 o} c_{B 0} \int_{0}^{1} C_{A 1}(f) C_{B}(f) \Phi(f) d f-$

$-k_{2 p} c_{B 0} \int_{0}^{1} C_{o R}(f) C_{B}(f) \Phi(f) d f$

with initial conditions: $\overline{C_{A 1}}(0)=1-\bar{f}, \overline{C_{A 2}}(0)=1-\bar{f}$ and $\overline{C_{p R}}(0)=\overline{C_{o R}}(0)=0$

where

$C_{A 1}=\frac{c_{A 1}}{c_{A 10}}, C_{A 2}=\frac{c_{A 2}}{c_{A 20}}, C_{B}=\frac{c_{B}}{c_{B 0}}, C_{p R}=\frac{c_{p R}}{c_{A 10}}, C_{o R}=\frac{c_{o R}}{c_{A 10}}$

Notice that this includes the effects of both chemical kinetics and mixing, with the mixing effect represented by the intensity of segregation $I_{s}$.

Concentrations versus the mixture fraction for the infinitely slow, instantaneous and intermediate rate reactions are given in Figure 3 a, b. Fig. 4 illustrates the effect of the mixing progress on the mixture fraction distribution, $\Phi(f)$. 
a)
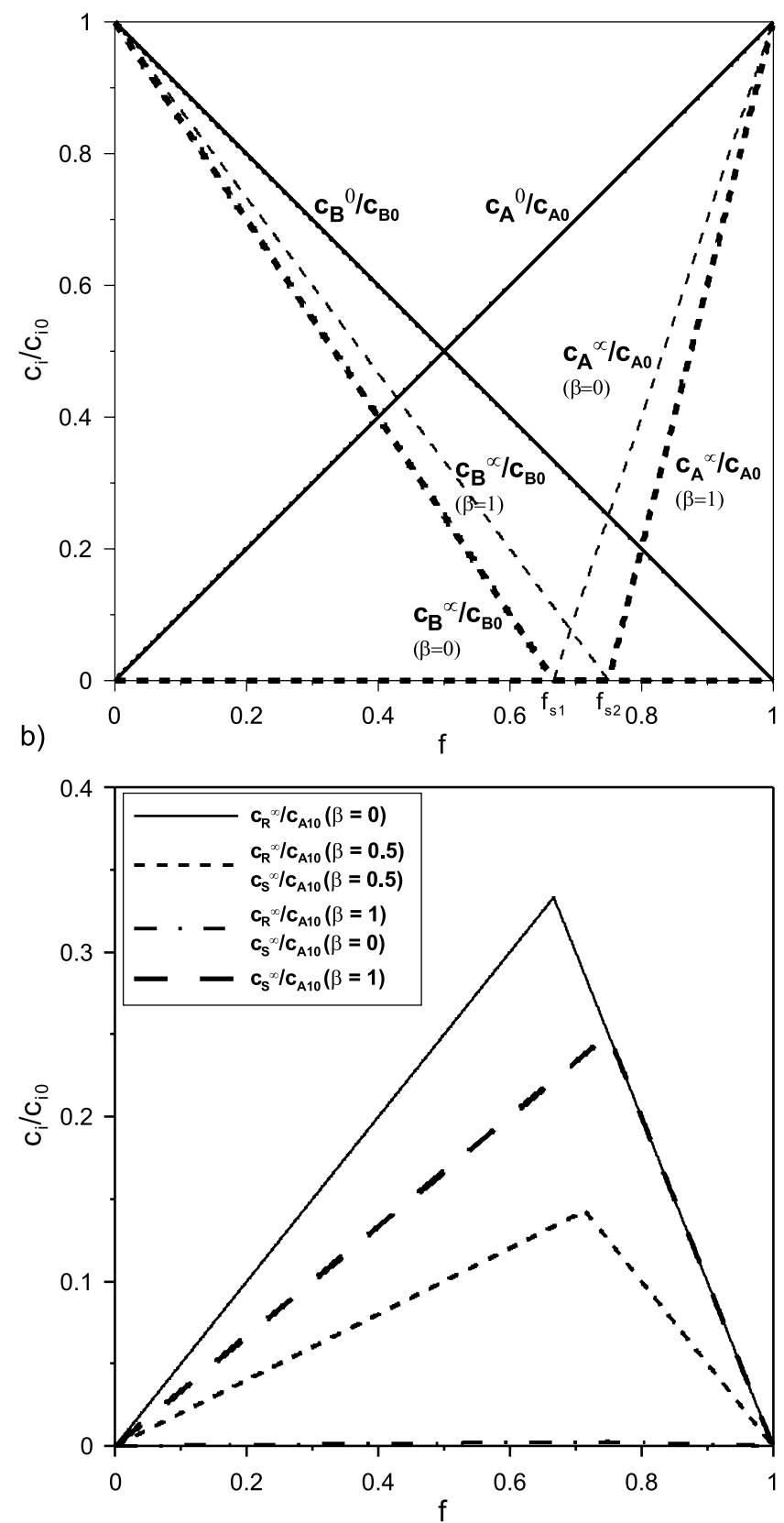

Figure 3. Concentrations v. mixture fraction for the infinitely slow and instantaneous reactions. (a) distributions of $c_{A}(f)$ and $c_{B}(f)$; (b) distributions of $c_{R}(f)$ and $\mathrm{c}_{\mathrm{S}}(\mathrm{f})$

The results of comparison between the PDF closure model and the E-model are shown in Fig. 5a. One can see that the engulfment model for the high volume ratio values gives the results not very different than those of the more complex closure model. However, the closure model can be directly linked to CFD. Fig. 5b shows the effect of the integral scale (the scale of energy containing eddies) on product distribution, illustrating in this way the effect of inertial-convective mixing, through the additional time constant $\tau_{S} \cong(1 / 2) \Lambda^{2 / 3} / \varepsilon^{1 / 3}$. One can see that with increasing the integral scale of turbulence both product distribution values increase. Notice that for $\Lambda \rightarrow 0$ the results reflect the process controlled by the viscous-convective effects, whereas for $\Lambda \rightarrow \infty$ the process is controlled by inertial-convective effects and hardly dependent on molecular properties, viscosity and diffusivity. a)

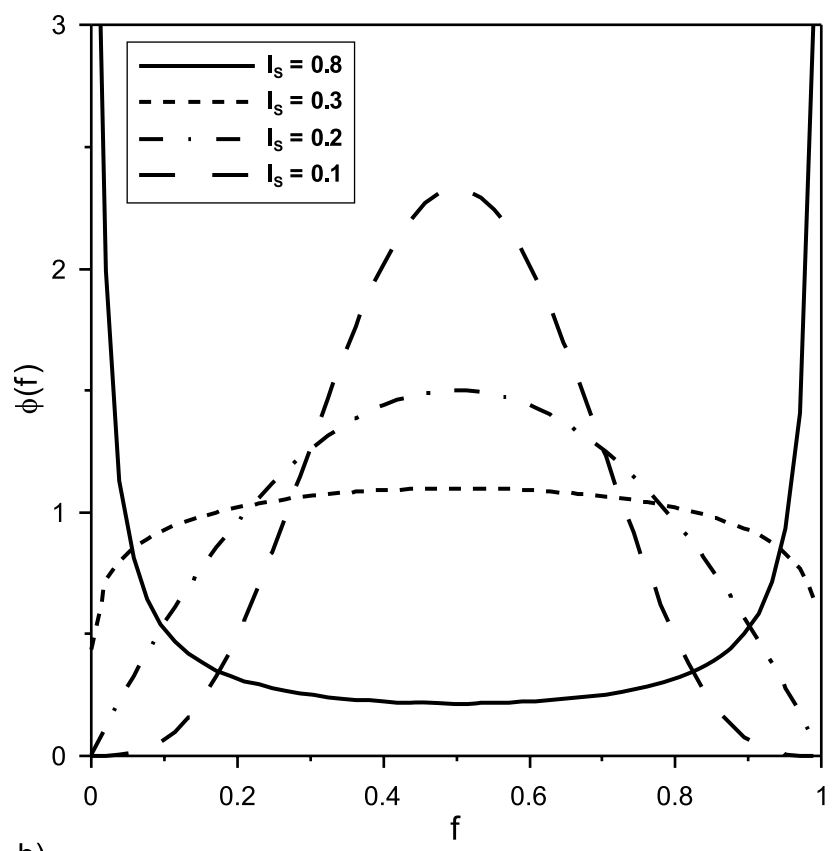

b)

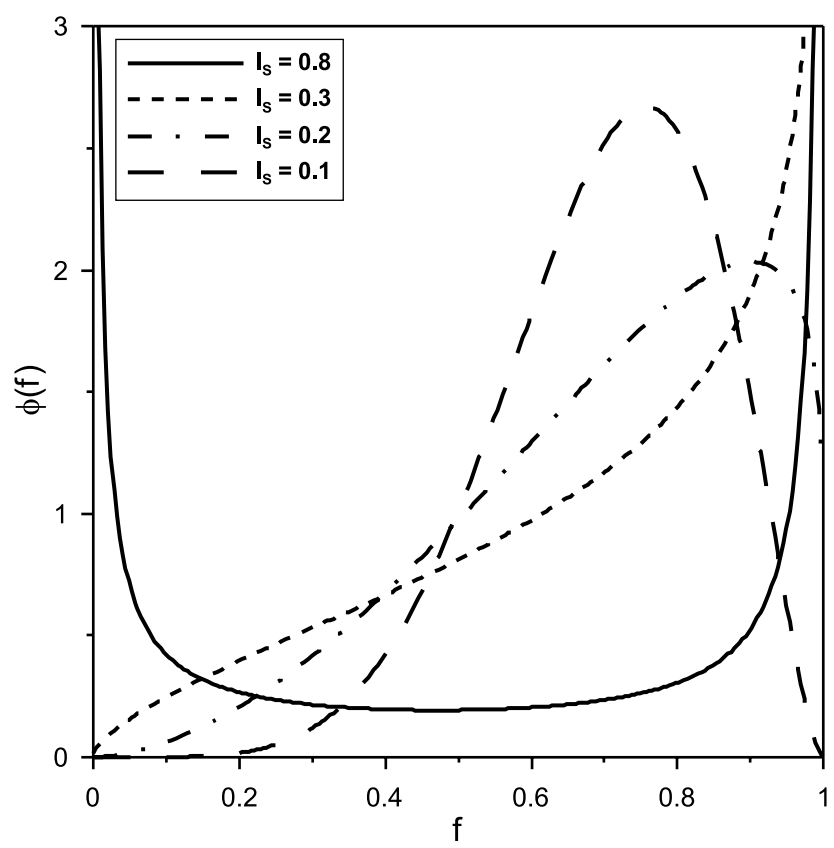

Figure 4. The probability density function (the beta distribution) for the mixture fraction. The effect of intensity of segregation on pdf. (a) $\bar{f}=0.5$; b) $\bar{f}=0.7$

\section{CONCLUSIONS}

A new method of closure for complex reaction schemes has been proposed and tested using simple flows and comparing model predictions for mechanistic modeling with the results of closure.

The presented results show also how to fit the test reaction system to the process conditions expressed by the rate of energy dissipation (power input) and the integral scale of turbulence (system scale effect).

\section{SYMBOLS}

$c_{i} \quad-$ molar concentration of reactant ,il,, $\mathrm{mol} \mathrm{m}^{-3}$

$\mathrm{C}_{\mathrm{i}}$ - dimensionless concentration of reactant , i", $\mathrm{C}_{\mathrm{i}}=\mathrm{c}_{\mathrm{i}} / \mathrm{c}_{\mathrm{i} 0}$, -

E - engulfment parameter, $\mathrm{s}^{-1}$

f - mixture fraction, - 
a)

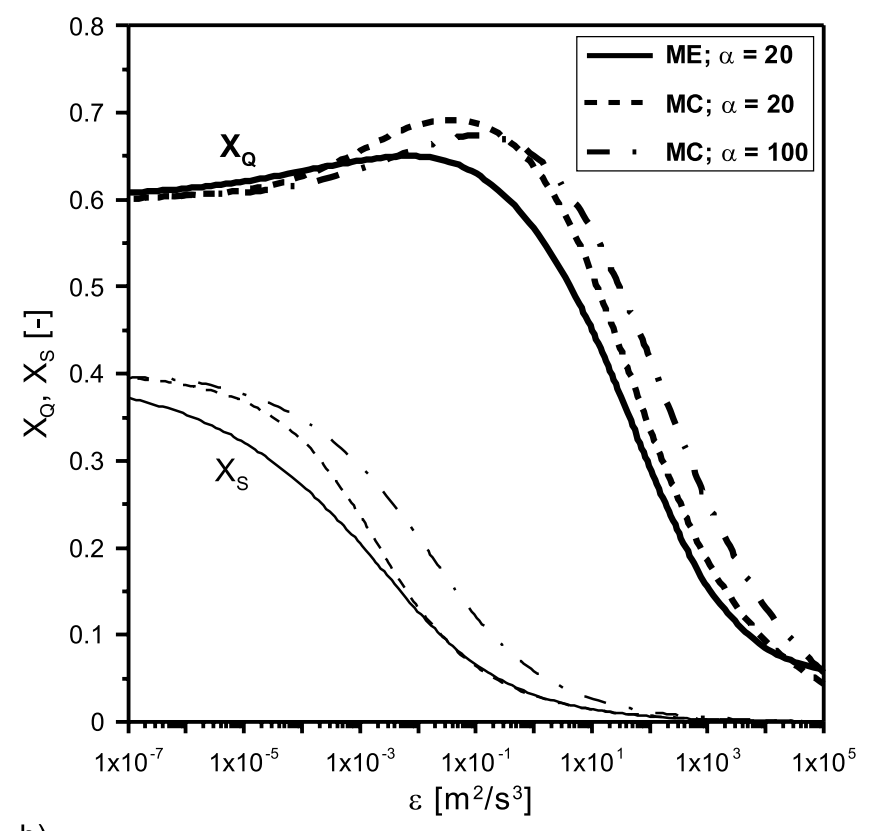

b)

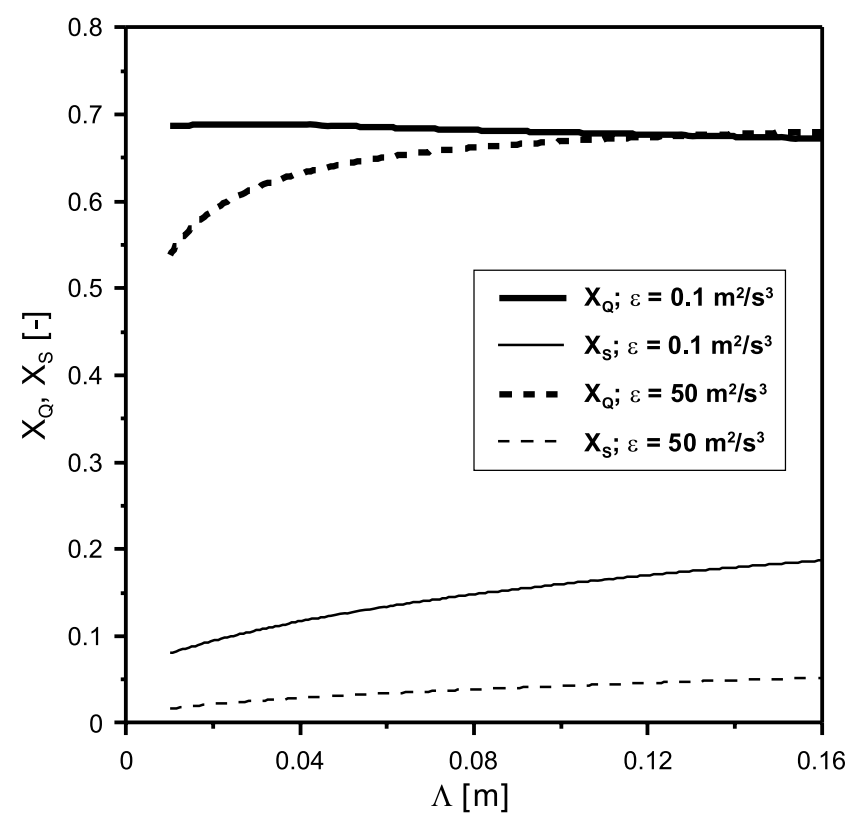

Figure 5. Predictions of PDF closure for $\mathrm{c}_{\mathrm{B} 0 \mathrm{sr}}=0.5 \mathrm{~mol} / \mathrm{m}^{3}$, $\mathrm{c}_{\mathrm{A} 10, \mathrm{sr}}=0.6 \mathrm{~mol} / \mathrm{m}^{3}, \xi=3$. (a) comparison of model predictions and the effect of volume fraction on product distribution, ME - Engulfment model; MC - Closure model; (b) Effect of integral scale of turbulence on the product distribution for the PDF closure

$\mathrm{c}_{\mathrm{i} 0, \mathrm{sr}} \quad$ - average feed concentration of ,i”

$\mathrm{I}_{\mathrm{S}} \quad$ - intensity of segregation, -

$\mathrm{k}_{\mathrm{i}} \quad$ - reaction rate constant, $\mathrm{m}^{3} \mathrm{~mol}^{-1} \mathrm{~s}^{-1}$

$\mathrm{N}_{\mathrm{i}} \quad$ - mole number of reactant ,i”, mol

$\mathrm{X}_{\mathrm{S}} \quad$ - yield of secondary product $\mathrm{S}$, -

$\mathrm{X}_{\mathrm{Q}} \quad$ - yield of competitive product $\mathrm{Q}$, -

\section{Greek}

$\alpha \quad$ - volume ratio, $\mathrm{V}_{\mathrm{A}} / \mathrm{V}_{\mathrm{B}}$, -

$\beta-$ parameter in eq. 17, 18, 19, -

$\varepsilon \quad-$ rate of kinetic energy dissipation, $\mathrm{m}^{2} \mathrm{~s}^{-3}$

$\gamma-$ stoichiometric ratio of 1-Naphtol to diazonium salt, -
$\Lambda \quad$ - integral scale of turbulence, -

$v \quad-$ kinematic viscosity, $\mathrm{m}^{2} \mathrm{~s}^{-1}$

$\tau_{\mathrm{s}} \quad$ - time constant of inertial-convective mixing, $\mathrm{s}$

$\xi$ - stoichiometric ratio of 2-Naphtol to 1-Naphtol, -

\section{Superscripts}

$0 \quad-$ means the infinitely slow reaction

$\infty \quad-$ means the instantaneous reaction

\section{LITERATURE CITED}

1. Bałdyga, J. \& Bourne, J.R. (1999). Turbulent mixing and chemical reactions. Chichester: Wiley.

2. Bałdyga, J., Henczka, M. \& Makowski, Ł. (2001). Effects of mixing on parallel chemical reactions in a continuousflow stirred-tank reactor. Trans IChemE. 79, 895 - 900. DOI: $10.1205 / 02638760152721109$

3. Bałdyga, J., Kowalski, A., Cooke, M. \& Jasińska, M. (2007). Investigations of micromixing in a rotor-stator mixer. Chemical and Proces Engineering. 28, 867 - 877.

4. Bourne, J.R., Kut, O.M., Lenzner, J. \& Maire, H. (1990). Kinetics of diazo coupling between 1-naphtol and diazotized sulphanilic acid. Ind. Eng. Chem. Res., 29, 1761 - 1765. DOI: $10.1021 / \mathrm{ie} 00105 \mathrm{a} 004$ 